\title{
LA DIMENSIÓN EDUCATIVA DE LA VIDA \\ DE RUFINO BLANCO Y SÁNCHEZ (1861-1936)
}

\author{
THE EDUCATIVE DIMENSION OF THE LIFE OF \\ RUFINO BLANCO Y SÁNCHEZ (1861-1936)
}

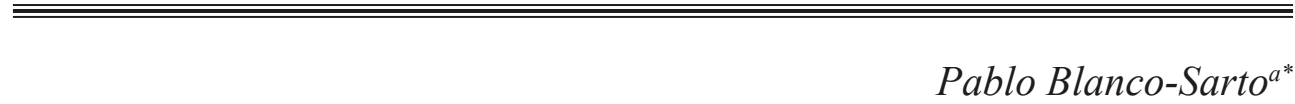

Fechas de recepción y aceptación: 28 de octubre de 2020 y 16 de marzo de 2021

DOI: https://doi.org/10.46583/edetania_2021.59.730

Resumen: Frente al ideal krausista sobre la cultura y la enseñanza, Rufino Blanco desarrolló una intensa actividad pedagógica y propuso un programa docente en la Escuela Superior de Magisterio de Madrid. Siendo autor regeneracionista de creencias cristianas, se opuso a la secularización de la vida pública y a la neutralidad religiosa en la escuela. Podría ser situado en la corriente católica, representada por Manjón, Ruiz Amado, Pedro Poveda y otros. De fundamentación neoescolástica, no dudó en dialogar con las corrientes pedagógicas de la época (Pestalozzi, Dewey, Mercier). En estas líneas se recoge un retrato pedagógico, intelectual y humano de este pedagogo español.

Palabras clave: regeneracionismo, renovación, escuela pública, escuela católica, periodismo.

Abstract: Faced with the Krausist ideal of culture and education, Rufino Blanco developed an intense pedagogical activity and proposed a teaching program at the Escuela Superior de Magisterio in Madrid. Being a regenerationist author of Christian beliefs, he opposed the secularization of public life and religious neutrality at school. It could thus be situated in the Catholic stream, represented by Manjón, Ruiz Amado, Pedro Poveda and others. With a neo-scholastic foundation, he did not hesitate to dialogue with the pedagogical currents of

${ }^{\text {a }}$ Departamento de Teoría e Historia de la Educación. Universidad de Navarra.

* Correspondencia: Universidad de Navarra. Facultad de Teología. Campus Universitario. 31009 Pamplona. España.

E-mail: pblanco@unav.es 
the time (Pestalozzi, Dewey, Mercier). In these lines a pedagogical, intellectual and human portrait of this Spanish pedagogue is collected.

Keywords: regenerationism, renovation, public school, Catholic school, journalism.

Rufino Blanco y Sánchez nació en la localidad alcarreña de Mantiel (Guadalajara) el 16 de noviembre de 1861, hijo de Julián Blanco y Pérez, practicante del pueblo, y Baltasara Sánchez y Alonso de Roa, madre y ama de casa. Siendo aún niño se trasladó con sus padres a Madrid, donde se diplomó en Magisterio. Fue maestro por oposición de la Escuela Superior de Niños de la Modelo-Municipal de Madrid (1889), donde creó el gabinete antropológico y posteriormente fue maestro regente -también por oposición- de la Escuela Práctica agregada a la Normal Central de Maestros (1894). Una vez casado en 1889, continuó sus estudios y se doctoró en Filosofía y Letras en la Universidad Central, donde fue discípulo predilecto de Marcelino Menéndez Pelayo (1856-1912). Durante muchos años fue profesor de la Escuela de Criminología, pero su vocación por la enseñanza le hizo centrarse en el campo de la pedagogía y de su historia, por lo que impartió docencia de esta materia en la Escuela Normal Central de Madrid. Al mismo tiempo, empezaba su trayectoria periodística escribiendo crónicas para diarios de América y Filipinas ya desde 1886 (cf. García González, 2008; Blanco Osborne, 2020). El propósito de estas líneas es un acercamiento bio-bibliográfico a partir de algunas primeras semblanzas ya publicadas, fuentes de la época y comentarios actuales, para poder abordar más adelante su obra y su pensamiento pedagógico. Estas páginas constituyen, pues, un punto de partida para ulteriores estudios pedagógicos. La metodología será sobre todo historiográfica, con algunas calas sobre las fuentes empleadas por el autor y el contenido de su pensamiento. Veremos así lo que se refiere en primer lugar a su labor educativa e investigadora, para abordar después su actividad pública, esbozar un breve perfil humano y reseñar de modo sumario su magisterio.

\section{Su Labor EDUCATiva}

La enseñanza primaria en España a finales del siglo XIX dejaba algo que desear. Tras el desastre de 1898 con la pérdida de las colonias de ultramar, su 
revitalización se había convertido en una de las banderas del regeneracionismo. Esta consistía en superar la postración del país con una buena educación. Rufino Blanco se mostraba plenamente partidario de este movimiento, y afrontó las deficiencias de la escuela pública implantando un sólido modelo organizativo: la escuela graduada, es decir, la escuela tal y como se concibe hoy. Este modo de organizar implicaba a los distintos niños en función de su edad, capacidad o -como sería más habitual- por el nivel de conocimientos. Proponía de igual manera una separación física en varias clases, asignando a cada una de ellas un maestro diferente bajo la supervisión de un maestro-director (cf. Garrido Palacios, 1979, pp. 89-95).

Ya en 1892, Blanco y Sánchez había presentado en la sección primera del Congreso Pedagógico Hispano-Portugués-Americano, para su aprobación, una moción con tres conclusiones: la constitución de grupos escolares, "la clasificación de los niños en tantas secciones como grados comprenda el programa de enseñanza" y el establecimiento de la "rotación de clases para todas las secciones del grupo escolar", es decir, que los maestros continuaran con los mismos niños, año tras año, desde el primero hasta el último grado. Ante su "sorpresa", las conclusiones fueron rechazadas con el voto en contra de sus "compañeros y amigos de siempre" (supuestos conservadores y maestros de escuelas públicas), mientras que el voto favorable venía de congresistas extranjeros y de "muchos adversarios en doctrinas pedagógicas" (institucionistas, liberales o republicanos). Esta actitud al margen de las ideologías marcará gran parte de su trayectoria profesional ${ }^{1}$.

También como regeneracionista y discípulo de Menéndez y Pelayo, Blanco reivindicó un lugar para la ciencia española, hasta el punto de demostrar -en una conferencia dada en Madrid- que la que se consideraba primera escuela

${ }^{1}$ En 1894, Rufino Blanco lideró una propuesta para convertir las escuelas normales de toda España en escuelas graduadas con -al menos- tres secciones, y la construcción de grupos escolares de acuerdo con el sistema panóptico. Dicha propuesta sería aceptada, en su primera petición, por un Real Decreto de 23 de septiembre de 1898 que convertía las escuelas anejas en graduadas de tres secciones con el regente como maestro-director y tres auxiliares. Esta sería la primera disposición legal, redactada por Rufino Blanco, que introducía en España, de forma tímida y a título de ensayo, el nuevo modelo organizativo. Además, se aventura en empresas relacionadas con la política educativa: con el marqués de Pidal (ministro de Fomento) en 1899, con el primer Gobierno de Silvela, con de La Cierva (ministro de Instrucción Pública en 1904), con Azcárraga y, posteriormente, con Fernández Villaverde y Cortezo (su ministro de Instrucción Pública en 1905) en sus planes reformistas de enseñanza. 
al aire libre fundada en 1903, en Charlottenburgo, fue posterior a la que ya en 1887 el padre Manjón había creado en Granada con el nombre de las Escuelas del Avemaría. En 1906, junto con Ezequiel Solana, impulsó y formó parte del equipo fundacional de la Sociedad Española de Pedagogía, y perteneció a la Federación de Amigos de la Enseñanza (FAE), fundada en 1930. De hecho, colaboró con numerosos artículos en la revista Atenas, órgano de difusión de este organismo.

Se doctoró en Filosofía y Letras en 1909 y fue nombrado ese mismo año, por el ministro de Instrucción Pública Faustino Rodríguez San Pedro, para crear -junto con otros compañeros- la Escuela de Estudios Superiores del Magisterio. En ella fue profesor numerario de Lengua y Literatura (1909). En esa institución llegó a ser profesor (1910-1931) y catedrático de Pedagogía Fundamental (1927), y desarrolló su programa docente sin abdicar de su fe católica a pesar de los tiempos ideológicos que corrían. Su doctrina educativa tiene gran influencia herbartiana y todo él está imbuido de un fuerte cristianismo de base tomista. En la línea de pensamiento del autor, resulta fácil encontrar la filosofía de Aristóteles y la tradición escolástica, reinterpretada por la Escuela de Lovaina, cuyo autor más representativo era el cardenal Mercier².

"Profundamente católico - afirma García González- diseñó su concepción pedagógica sobre el armazón de la filosofía escolástica fundamentando la educación en la religión, sin apartarse un ápice del pensamiento católico". Frente a la reserva religiosa de los innovadores, Rufino Blanco se opone a esa secularización de la vida pública y a la neutralidad religiosa en las aulas, aunque su claridad de postura no se tradujo nunca en una actitud agresiva ni polémica. La Escuela de Estudios Superiores estuvo dotada de un excepcional elenco docente de distintas orientaciones religiosas e ideológicas entre los que

${ }^{2}$ Su fidelidad a la tradición aristotélica no le impidió entrar en las concretas orientaciones metodológicas modernas y aceptar las entonces innovadoras orientaciones pedagógicas de Froebel y Pestalozzi, hasta llegar al padre Manjón, con el que mantuvo una estrecha relación de amistad. Blanco y Sánchez coincide en su ideología con los también pedagogos españoles Ramón Ruiz Amado y el padre Poveda. Blanco manifiesta siempre su condición de católico, haciendo suyas unas palabras de su maestro Menéndez Pelayo: "Católico soy y como católico afirmo la providencia, la revelación, el libre albedrío, la ley moral, bases de toda historia. Y si, al juzgar ideas, tropiezo con algunas que pugnan con las mías, ¿qué he de hacer sino condenarlas? En reglas de lógica y en ley de hombre honrado y creyente sincero tengo obligación de hacerlo". 
destacaron, además de Rufino Blanco, los profesores José Ortega y Gasset, Luis de Zulueta, Domingo Barnés y Juan Zaragüeta, entre otros ${ }^{3}$. Blanco se interesó igualmente por la educación femenina, la educación sexual, la coeducación, la higiene escolar, la importancia de la educación física y el valor pedagógico de los juegos. Incluso llegó a intervenir en elementos de mobiliario escolar implantando un nuevo tipo de mesas para escribir de pie, siguiendo el modelo alemán de la época.

Como profesor de la Escuela Superior de Magisterio realizó una importante labor en el terreno de la bibliografía sobre educación. "Rufino Blanco -escribe Isabel Corts-, comprometido en la tarea educativa, quiere escribir una teoría de la educación que recoja las aportaciones válidas que se han ido elaborando a través del tiempo, y para ello, como paso preliminar, ve necesaria la revisión de las obras pedagógicas publicadas hasta entonces" (cf. Corts Giner, 1986, p. 53). Representa el contrapunto neocatólico a la pedagogía de la Institución Libre de Enseñanza de Giner, Cossío y muchos otros. Así, como maestro y formador de maestros creó el Gabinete Antropométrico de la Escuela Modelo de Madrid, introdujo la caligrafía vertical, colaboró en la implantación de las escuelas graduadas, realizó innovaciones en numerosos aspectos de la organización escolar y se interesó por los temas anteriormente mencionados. Por ejemplo, sostiene Xavier Torrebadella-Flix, de la Universidad Autónoma Barcelona: "Rufino Blanco y Sánchez materializó una de las mayores contribuciones pedagógicas, académicas y científicas de la educación física contemporánea" (Torrebadella-Flix, 2014, p. 281).

\section{SU LABOR INVESTIGADORA}

Pongamos tan solo un ejemplo. Como Menéndez y Pelayo, erudito e investigador, escribió una Bibliografía pedagógica de obras escritas en castellano o traducidas a este idioma. Contiene este trabajo tres mil setecientas setenta fichas de libros procedentes de España y Latinoamérica. Así, por ejemplo, del

${ }^{3}$ García González (2008, p. 3). Otros nombres aparecidos son Eugenio Piñerúa, Pablo Martínez Strong, Ángel Vegue y Goldoni, Ricardo Beltrán y Rózpide, Luis de Hoyos, Francisco de las Barras Aragón, Magdalena Fuentes y Anselmo González. 
siglo XIII se reseñan 2 obras pedagógicas, dieciséis del siglo XIV, 12 del siglo XV, 90 del siglo XVI, 107 del siglo XVII, 307 del siglo XVIII, 1914 del siglo XIX y 1154 solo de la primera década del siglo xx. Esta obra, en la que empleó quince años de su vida, resulta en su conjunto un instrumento de trabajo verdaderamente indispensable para todo el que se interese por la historia de la educación española (cf. García González, 2008, pp. 1-4) .

En la redacción de esta Bibliografía pedagógica fue dirigido por su insigne maestro y polígrafo Menéndez Pelayo, a quien dedicó la obra. Los trabajos bibliográficos de Rufino Blanco tienen un carácter propedéutico y fundamental, como actividades previas y necesarias para un quehacer rigurosamente científico, en el que no solo se vierten sus propias ideas, sino que en ellas van incluidos determinados contenidos culturales que persisten a lo largo del tiempo. Así, Rufino Blanco publicó en total 57 obras, 40 de ellas didácticas, de entre las cuales han tenido especial difusión su mencionada Bibliografía pedagógica del siglo $X X$ (traducida en las principales lenguas europeas y editada en España por suscripción popular), una Bibliografía general de Educación Física, una Teoría de la Educación, otra Teoría de la enseñanza, un Tratado elemental de Pedagogía y una Organización escolar.

Actualmente puede decirse que merecen especial consideración sus contribuciones al ámbito filológico en un sentido amplio, en particular su Tratado de Análisis de la Lengua Castellana (1909), modelo de exposición metodológica en una tradición que probablemente culmina allí y que es desgraciadamente interrumpida, así como el Arte de la Lectura, publicado por primera vez en 1894 y que en 1927 había alcanzado su decimoprimera edición. Esta obra cons-

\footnotetext{
${ }^{4}$ Las notas a los artículos son modelo de propiedad, concisión y claridad, además de estar realizadas con un criterio básicamente orientador. Todo el material bibliográfico fue revisado, cuando no confeccionado, por Rufino Blanco, venciendo obstáculos casi insuperables con una constancia y dedicación verdaderamente ejemplares. La abundancia de la cosecha compensaba a menudo el trabajo: otras veces el examen de cinco mil papeletas no dio sino dos notas útiles para esta Bibliografía. Sobre ella nuestro autor escribió: "Hubo libro que fue buscado en vano en todas las bibliotecas públicas de España y en dieciséis del extranjero, y hasta la resistencia física hubo que poner a prueba pasando veintidós mil papeletas de un índice bajo la temperatura de 38 grados o copiando manuscritos en el Alcázar de Segovia en un ambiente de 5 grados bajo cero. Era esta empresa, en definitiva, superior a las fuerzas de una persona, $\mathrm{y}$, sobre todo, de un hombre que tiene mermada su actividad por otras incesantes ocupaciones. Pero el temor de no llegar a la perfección no debía retraerme de llevar a cabo el trabajo posible y he preservado en él hasta el límite máximo del tiempo concedido oficialmente para realizar el empeño".
} 
tituye, tanto por su amplitud teórica de criterios, más allá del núcleo técnico de la lectura en voz alta, como por su concepto de eficacia práctica y extensión, el tratado fundamental y más influyente de este género retórico en lengua española. También son dignos de consideración unos Elementos de literatura española e hispanoamericana que, en 1925, ya iban por su tercera edición ${ }^{5}$.

\section{Su ACTIVIDAD PÚBLICA}

Junto a la vocación docente, apareció el periodismo: fue ya en 1889 redactor de La Enseñanza de Madrid y copropietario y redactor entre 1896 y 1902 de El Magisterio Español (1896-1902), junto con Victoriano Fernández Ascarza y Ezequiel Solana. Desde 1902 dirigió el diario católico El Universo (19091926), que fundó junto con Juan Manuel Ortí y Lara, el marqués de Comillas, y el periodista y escritor democristiano Álvaro López Núñez. De igual manera codirigió La Educación Hispanoamericana y fue colaborador asiduo de $A B C$, en donde firmó unas veces con su nombre y otras bajo el seudónimo Un Crítico de la Alcarria, además de presidente honorario de la Federación de Asociaciones de Prensa de España desde que se creó en 1922 hasta 1926, compartiendo este puesto con el presidente honorario de esta, José Francos Rodríguez, y vicepresidente de la Asociación de la Prensa de Madrid (1920-1926).

El 29 de marzo de 1936, ocupó la vacante del político español José Sánchez Guerra en la Academia de Ciencias Morales y Políticas, presidiendo el acto de recepción de su ingreso el presidente de la República, Niceto Alcalá-Zamora. Su discurso versó sobre los fundamentos de educación moral y cívica. Para él la educación moral equivale a la educación de la voluntad, y sobre esta idea expone su pensamiento acerca de los grandes problemas de la vida, como la

\footnotetext{
${ }^{5}$ Encontramos también otras obras como Apuntes sobre Biología pedagógica, Paidología y Pidotecnia; Ideas de educación física del pueblo griego, según Philostrato "Peri Gymnastikees"; Quintiliano y sus sistema de educación; Luis Vives, la Pedagogía cientifica y la Instrucción de la mujer cristiana; Rollin y el Tratado de los estudios y una serie de monografías entre las que destacan las dedicadas a Platón, Pestalozzi, La Salle y Quintana. También redactó los capítulos de pedagogía contenidos en la monumental Enciclopedia Espasa-Calpe. Varias de estas obras fueron editadas con cargo al Estado y premiadas por la Real Academia Española y la Biblioteca Nacional, así como en diversos congresos y exposiciones nacionales e internacionales (París, 1900; Bilbao, 1905; Quito, 1909 y 1910).
} 
educación, la libertad, el placer y el dolor. Mostró entonces la propia erudición de sus trabajos y expresó con claridad su juicio acerca de los problemas que planteaba. Blanco se opuso firmemente a la idea "de que la energía y la perseverancia en el proceder no dependen de la voluntad”. Según él, esta creencia en "la fuerza de voluntad es solamente una manera de decir: es una expresión puramente tropológica en que se toma el efecto por la causa" (Blanco y Sánchez, 1939) ${ }^{6}$. Dividida en dos bandos durante la guerra civil, la Academia perdió a una tercera parte de sus miembros entre las bajas durante el conflicto -entre los que se encontraba él-y la depuración de académicos republicanos, ya en la inmediata posguerra (cf. Martorell Linares, 2002, pp. 229-252, especialmente pp. 229 y 234$)^{7}$.

En 1920 había sido elegido vicepresidente primero de la junta directiva de la Asociación de la Prensa de Madrid y, desde 1921, fue consejero de Instrucción Pública. En sus múltiples viajes conoció el movimiento pedagógico moderno preconizado por Claparéde, Binet, Kerschensteiner, Dewey, el cardenal Mercier y tantos otros con los que mantenía relaciones profesionales. Fue nombrado para regir el Instituto Normal de Filosofía de La Paz (Bolivia)

${ }^{6}$ El autor presentó así una clara oposición a la moral sentimental que personifica Schopenhauer, afirmando con claridad que la voluntad es una facultad racional, cuya educación tiene como finalidad la formación de hábitos. Así se evitaba todo posible voluntarismo. Según Blanco, solo algún autor despistado podría decir algo, como Rousseau, de que "el único hábito que se debe dejar tomar al niño es el de no contraer ninguno", pero esto no pasa de una frase de un hombre que no carecía de hábitos, aunque no todos eran recomendables. Terminó con las siguientes palabras: "De todas suertes, espero que el académico que acepte la carga de contestar a mi discurso suplirá con menos palabras y con abundancia de doctrina los vacíos de mi pobrecilla disertación que yo titularía «el canto del cisne», si yo fuera cisne y si los cisnes cantaran. Yo no soy más que un maestro de escuela y a lo más, a lo más «un crítico de la Alcarria». He dicho".

${ }^{7}$ Como decíamos, en 1909 había sido comisionado con otros compañeros por el entonces ministro de Instrucción Pública, don Faustino Rodríguez-Sampedro, para crear y organizar una Escuela de Estudios Superiores de Magisterio, equivalente a una Facultad de Pedagogía, pero con su propia autonomía y libertad de acción, pues estaba presidida por un comisario regio que dependía directamente del ministro. En 1914 apareció el primer Plan de estudios y, unido al plan ministerial de creación y construcción de escuelas, creó en España un nuevo estilo de Magisterio Nacional, bien formado y dirigido. Rufino Blanco defendió en la escuela primaria la idea de la escuela graduada, cuyo nuevo sistema apareció en la primera decena del siglo xx, y con un mínimo de esfuerzo se conseguiría un máximo rendimiento. Fue miembro de la Junta para la Extinción del Analfabetismo y de la comisión Interministerial de educación física. Con esto fue introduciéndose en la vida pública del país, especialmente en lo referido a la cultura y la educación. 
en 1916 (cf. Barrera, 1916), por lo que llegó a conocer en sus múltiples viajes el movimiento pedagógico moderno promovido por los mencionados autores. Cuando en 1927 obtuvo la cátedra de la Escuela de Estudios Superiores de Magisterio por oposición, pronunció una conferencia sobre Menéndez Pelayo, bibliógrafo y erudito. Fue además condecorado y ocupó cargos relevantes en instituciones culturales y políticas ${ }^{8}$.

Cronología de algunas publicaciones de Rufino Blanco

1885 Método para aprender la letra española y Método para aprender la letra inglesa.

1906 Tratado elemental de pedagogía.

1907 Método de lectura y escritura. Segunda Parte del Primer Grado y Método de lectura y escritura. Tercera Parte del Primer Grado.

1907-1912 Bibliografia pedagógica.

1909 Pestalozzi su vida y sus obras. Pestalozzi en España.

1910 Arte de la Escritura y de la Caligrafia Española.

1912 Teoría de la educación.

1914 Una vista al magisterio católico en el Monasterio de Piedra.

1916 Método de lectura y escritura. Primer Grado. Primera Parte.

1917 La Misión Docente.

1920 Refranero Pedagógico hispanoamericano.

1922 Notas bibliográficas referentes a la historia de la educación y a la historia de la pedagogía.

1925 Don Marcelino Menéndez y Pelayo. Apuntes Bibliográficos.

1925b Nociones de lengua castellana o española.

1927a Bibliografía general de la educación física: con datos copiosos referentes a la biología, antropología, anatomía, fisiología, higiene, eugénica, puericultura, paidología, pedagogía y otras ciencias fundamentales del ejercicio corporal educativo.

\footnotetext{
${ }^{8}$ Hombre de confianza del Claudio López Bru, marqués de Comillas, durante la dictadura de Primo de Rivera había sido nombrado también concejal del Ayuntamiento de Madrid desde abril de 1924, donde abogará a favor de una reforma de la enseñanza pública. Desempeñó en fin también el cargo de gobernador civil de la provincia de Segovia: nombrado en agosto de 1927, cesó en el cargo al poco de comenzar la llamada "dictablanda" de Dámaso Berenguer.
} 
1927b Los problemas de la educación física. Preguntas incontestables. La Escuela Moderna, vol. 432, pp. 393-395.

1927c Menéndez y Pelayo como bibliógrafo y erudito.

1928a Los problemas de la educación física. La Escuela Moderna, vol. 436, pp. 1-4.

1928b Los problemas de la educación física. La Escuela Moderna, vol. 438, pp. 9799.

1928c Método de lectura y escritura. Primer Grado. Primera Parte.

1929 Notas bibliográficas correspondientes a la conferencia sobre educación física en la escuela primaria.

1930 Teoría de la educación, t. I (2. a ed.).

1930b Congreso Nacional de Educación Primaria y Normal.

1931a La Separación de la Iglesia y el Estado.

1931b La educación de la mujer y la coeducación de los sexos.

1932a Ideas de la educación física del pueblo griego según Philostrato. "Peri Gymnastikees" (2. ${ }^{\mathrm{a}}$ ed.).

1932b Método de lectura y escritura. Primer Grado. Segunda Parte.

1931-1934 Notas sobre el movimiento pedagógico.

1934 Pedagogía. Enciclopedia Universal Ilustrada Euro-Americana.

1935 Congresos, asambleas y otras reuniones públicas de carácter pedagógico.

1936a Bibliografía pedagógica española 1926-1935.

1936b La República y la Legislación vigente de la Enseñanza Privada. La clausura de escuelas y el Código Penal.

1936c España y la pedagogía. Conferencia en Unión Radio.

1936d Memoria de "El Universo" en la Exposición Mundial de Prensa Católica celebrada en el Vaticano en mayo 1936.

1941 Tratado Elemental de Pedagogía.

\section{SU PERFIL HUMANO}

Una semblanza (Zahonero, 1916) presentaba a don Rufino como una persona juiciosa, y lo definía como un "hombre en el cual la corrección es inalterable[,] la consistencia mental segura", ya que "ni en su cerebro se desvía el raciocinio por seducciones de la imaginación", sino que se guía "por la lógica regularidad del discurso", si bien esto no le impide "lucir un estilo galano y bellezas de estilo". De igual manera lo describía como "un trabajador admirable", hasta el punto de que escribía el comentador: “¿Cuándo hace el Sr. D. Rufino Blanco 
todo lo que hace? ¿Qué tiempo le queda al maestro, que jamás falta a su cátedra, para dirigir trabajos de un periódico diario y al católico de acción de acudir a juntas, consejos, conferencias y devociones, y luego pueda ser el hombre de sociedad, correctísimo en cortesía y exacto en sus deberes, para ser minero afanado en la investigación de biblioteca, erudito informador y autor de obras de innegable utilidad y mérito?".

En un panegírico (Getino, 1917) ensalzaba "el crédito ensalzado, inmortal, del inmaculado color de su apellido". Y tras recorrer los mencionados méritos académicos, exclamaba entusiasta el comentador con finura psicológica:

Miradlo si podéis pues no se deja ver más que un emperador de la China, enfrascado siempre en sus tareas científicas: ágil, pulcro, delgado de modales finísimos, sutil y natural en su conversación, que es condición rarísima de ingenios; sus ojos escrutadores se posan melancólicamente en su interlocutor [...]; su voz tenue, insinuante, fluye segura en un inagotable ideario personal que él presenta cual si solo tratara de sugerir lo que enseña; todo en él es transparente, noble, acariciador.

A lo que añade la nota de la discreción por medio del siguiente pasaje descriptivo:

Hay que ir para ver, a su hotel de Chamartín, a su clase, o a la redacción de $E l$ Universo, periódico que él dirige hace mucho, y que es el más moderado y el menos personal de todos los periódicos, español y católico nada más, como su director. Encontrar a don Rufino en paseos, tertulias y teatros me parece del todo imposible. Así ha podido realizar, a los cincuenta y cinco años, una obra de titanes.

En una nota se recogía el homenaje con motivo de su jubilación (Homenaje, 1932), aparecían sus conocidos y reconocidos méritos profesionales, a lo que añadía de modo elocuente: "pero hay en la vida de Rufino Blanco una realidad que transciende mucho más de lo que su propia modestia puede limitar, y es la influencia de la labor benemérita a través de cuarenta y cinco años de constante y diaria actuación". Es decir, se valoraba su oculta labor que, con el paso del tiempo, había sido eficaz y luminosa. La cotidianiedad y la constancia eran así su principal virtud, que solo harán constar sus frutos mucho después. Un 
año después, aparecía un documento (Rogerio Sánchez et al., 1933) en que se hallaba un total de 644 "discípulos, compañeros y amigos suyos" de todo el territorio nacional que "quisieron conmemorar con público homenaje la fecha del 16 de noviembre de 1931, fecha en la cual se quería firmar el decreto de jubilación, pero la indeclinable labor de este hizo inútil todo proyecto".

El Boletín de la Institución teresiana (1935, p. 115) se unía a los parabienes con motivo de su ingreso en la Academia de Ciencias morales y políticas. "Lo que significa para la Institución Teresiana, donde tanto agradecimiento, veneración y cariño se profesa por el señor Blanco, no es necesario subrayarlo. Habiendo seguido tan de cerca la vida de nuestra obra...", explicaban que "compartamos sus sentimientos y celebremos su triunfo". Aludía después a una vida dedicada a la investigación y la enseñanza, "campos en los cuales su labor ha marcado una huella profunda, fertilísima, definida y matizada siempre por sus arraigadas convicciones religiosas, que son el móvil de todas sus actuaciones". "En su noble figura de caballero cristiano está la clave y la explicación de toda su vida".

En otro homenaje (Vida Económica, 1935, p. 125) añadía en lenguaje colorido y pintoresco: "no menos de 80 volúmenes ha escrito, alternando la construcción de esta obra ingente con la del periodismo, del que Rufino Blanco es gran maestro, y la de literato, y la de esgrimidor de una pluma de oro por lo valiosa y de acero por lo recia contra los malandrines y follones que ofenden al glorioso idioma de Cervantes". A pesar de su extremada delgadez, consecuencia de un régimen vegetariano, disfrutaba de envidiable salud y nunca en su vida estuvo enfermo. Falleció fusilado al poco de comenzar la Guerra Civil española, el 2 o el 3 de octubre de 1936, en Madrid, víctima de la represión en zona republicana. Según testigos presenciales, podría decirse que murió mártir de la fe y la cultura, las cuales él siempre consideró inseparables y que constituían una clave de su humanismo ${ }^{10}$.

${ }^{9}$ La conmemoración consistió en una edición actualizada de la Bibliografía pedagógica del siglo $X X$, de modo que simplemente recoge este documento las adhesiones, encabezadas por José Rogerio Sánchez, secretario, y Antonio Domingo Calderón, tesorero.

${ }^{10}$ El propio general Francisco Franco, como respuesta a una entrevista para el diario La Prensa de Buenos Aires, reproducida en 1938 en el $A B C$ de Sevilla, declaró: "esa gente [=los republicanos] no habla de cómo fueron asesinados fríamente, con saña que pone espanto en el ánimo más templado, don José Calvo Sotelo, don Víctor Pradera, don José Polo Benito, el duque de Canalejas, don Honorio 
Figuran 324 firmas en las que se afirma que padre e hijo fueron fusilados el 3 de octubre. Las notas necrológicas recorren las radios y periódicos de la época. El sábado 28 de octubre se hacía eco, durante media hora, la Radio oficial del Estado con sede en Burgos, y al día siguiente la Radio local de Huesca reseñaba que el "notable pedagogo cristiano y conocido publicista" don Rufino Blanco había sido "muerto por los rojos". También El Noticiero de Zaragoza de ese mismo día indicaba, en una crónica firmada en Pamplona, que había sido "vilmente asesinado" el "pedagogo quizás más autorizado de España". Invitaba a toda España a aprovechar "su lección sabia" "iy sobre todo su sangre mártir!". "Pocos hombres han hecho -concluía- tanto bien en su vida privada y en su vida pública". Figuran también reseñas en los zaragozanos Heraldo de Aragón y el Amanecer, el cual alude a su vez a "sendas crónicas" publicadas en Navarra. El Diario vasco del primero de diciembre de 1936 reseñaba su "figura venerable y patriarcal, su afabilidad, su deseo de ser útil a sus infinitos consultantes". De nuevo, el Heraldo de Aragón del 3 de diciembre lo mencionaba como "triunfante en el reino de la Luz", mientras que dos días después señalaba como "delito" "su valor y caballerosidad y amor a Dios y a España"

Maura, don Francisco Valdés, don Rufino Blanco, don Manuel Bueno, don José María Albiñana; don Ramiro de Maeztu, don Pedro Muñoz Seca, don Pedro Mourlane Michelena, don Antonio Bermúdez Cañete, don Rafael Salazar Alonso, don Alfonso Rodríguez Santamaría, presidente de la Asociación de la Prensa; don Melquíades Álvarez, don Enrique Estévez Ortega, don Federico Salmón, padre Zacarías G. Villadas, don Fernando de la Quadra, don Gregorio de Balparda y tantos otros cuya lista haría interminable estas contestaciones. Queda dicho que no hemos fusilado a ningún poeta" (sic; cursiva nuestra).

${ }^{11}$ De ese mismo año de 1936 se conserva también el acta de incautación de tres colchones por parte de agentes afectos a la Comisaría de Chamberí, y fechada el 23 de octubre de 1936. Constan también en los archivos de la Academia de ciencias morales y políticas el acuerdo de la Comisión de la Excelentísima Diputación de Huesca (17/12/1936), respectivas necrológicas con la retórica de la época del Faro de Vigo (25/11/1936), del Diario regional de Valladolid y del Diario de Burgos (4/12/1936) -con referencia al día siguiente al funeral celebrado en la catedral-y de la Hoja oficial del lunes de Zaragoza (14/12/1936), unas reseñas del funeral y de un homenaje de la Escuela normal, aparecidas en $E l$ Noticiero, El Heraldo de Aragón y en el Amanecer de la misma ciudad (15/12/1936), así como en el Diario vasco. Del 30 de diciembre del Diario de Huesca. El $A B C$ descubrirá una lápida en honor de los periodistas fallecidos el 22 de agosto de 1939, entre los que figuran Julián y Rufino Blanco. 


\section{Su MAGisterio}

Un acto realizado en Zaragoza en enero de 1937 fue titulado significativamente "Homenaje a un pedagogo mártir". Allí, el señor Gómez Lafuente lo calificaba como modesto y "un dechado perfecto de educación y humildad". Y continuaba con las siguientes palabras: " $\mathrm{Si}$-como dice Gracián- es dichoso un hombre con tres «eses», por santo, sabio y sano, Blanco fue un hombre sumamente dichoso". En un artículo de José Muntada Bach, redactado en Zaragoza en febrero de 1937, destacaba que: "Las virtudes del maestro eran laboriosidad, puntualidad, sencillez que inspiraba respeto, método, y nítida alma española, y todo ello articulado solo con lo aprovechable y adecuado de la cultura de fuera". Por su parte, Prudencio Landín lo definía como "hombre fervorosamente cristiano", que "impregnó todas sus obras con este perfume espiritual, sin cerrar paso a estas ideas innovadoras. [...] No escamotea ninguna idea a sus discípulos y lectores. Tiene la lealtad de poner ante sus ojos todo el cuadro de la vida pedagógica del mundo, pero pensando siempre en España y en las peculiaridades de nuestra raza" (Boletín de Educación de Zaragoza, 1937, p. 19; Boletín provincial de Educación de Pontevedra, 1937, pp. 15 y 16).

En una nota del 14 de marzo de 1940, se permite trasladar los restos mortales del Cementerio de La Almudena a la Sacramental de San Justo y San Pastor, con una exención de derechos de traslado del cadáver. En la necrológica de Juan Zaragüeta (1961, pp. 243-252), “académico y secretario perpetuo" de la Real Academia de ciencias morales y políticas, fue presentado -junto con Álvaro López Núñez y Ramiro de Maeztu- como "apóstol y mártir de los ideales triunfantes en la guerra de 1936 a 1939”. Debe quedar clara su afiliación política, probablemente distinta a la predominante en aquellas fechas de 1961. Allí menciona su muerte tras "un interrogatorio en el que sin duda se ratificó en los ideales de toda su vida y por los que la sacrificó". Destacaba así el discípulo su coherencia y su unidad de vida: "Pocas personas hay en que se dé un acuerdo tan perfecto de una vida encarnada en una doctrina y una doctrina encarnando una vida". A esto añadía que la suya era "un modelo de fidelidad a una vocación": la enseñanza primaria. A esto añadía una caracterización de su docencia: "asiduidad en la asistencia a clase, esmero en la preparación, claridad, orden y método en la preparación magistral”. Como criterios definitorios de su ideario pedagógico - que desarrolla a continuación- 
figuran la "amplitud de criterio, ponderación de juicio y espíritu progresivo", entendido como "amplitud de criterio", por la amplitud de información sobre la "pedagogía vigente y antigua".

En un artículo de homenaje de Manuel Navarro de ese mismo año (1961) destacaba sus "dulces maneras" y una "humildad sincera", y vuelven a ser mencionados sus "cuarenta y cinco años de su conducta y de su diaria actuación". En su lugar, rechazó un homenaje público y propuso que "cerca de un millar de españoles costeamos una tirada especial de sus obras". Y añade: "Las puertas de su casa estaban abiertas a los discípulos y amigos que acudían a él para consultar sus dudas e incluso contarle sus cuitas". Destaca además cómo su esposa, tras la detención de su marido junto a su hijo, "casi ciega, sobreponiéndose al dolor de esposa y de madre, acudía todos los días a la puerta de la checa, para llevarles algo de comer y ofrecerles sus oraciones y su cariño inmenso". Su hijo Ramón recoge en un artículo la anécdota del blasón de los Blanco ya mencionada (1961). Recoge también un trabajo que asumió don Rufino tras su jubilación, quien "con otros «héroes»" colaboraban "para contrarrestar la enseñanza oficial que había suprimido el catecismo en las escuelas primarias. En más de 150 llegó a tener «cruzados», las cuales [escuelas] el Gobierno republicano clausuraba con el menor pretexto". Termina este recuerdo con una simpática anécdota sobre su reacción ("que le hizo reír a carcajada limpia") ante una denuncia por un falso y supuesto plagio. Al parecer, el sentido del humor le acompañaba también en su atareada vida.

En otro homenaje del mismo año (Arias Corrales, Aguirre Prado, Pérez Darío-Zori, 1961), Luis Aguirre Prado añade -a las virtudes ya mencionadas-el ser "fiel a sí mismo", "sin que quebranten esa fidelidad ni halagos ni desalientos, ni deslealtades ni honores". La finalidad de sus obras sería "mover a las voluntades a hacer el bien". "Claridad expositiva y mantenimiento de la verdad en todo instante, mostrando lo pernicioso de todo falseamiento. Conducta y obra en consonancia. Excedido en virtudes don Rufino, y por tanto inmaculado de ruindades, fue constante su ataque al error, la apología de la pureza doctrinal. Ni la más liviana concesión a cuanto se generara a desviaciones de la suprema enseñanza [...d] el amor. Para la proclamación y el mantenimiento de la verdad trocaba en cátedra cualquier elemento que garantizase la eficacia", sea la conferencia o el artículo periodístico. Virgilio Pérez reseña "su férrea voluntad, su incansable laborar, su vocación profesional, su espíritu españolísimo, 
su absoluta voluntad de llegar a la meta propuesta". Por su parte, Darío Zori da noticia de su última conversación en verano de 1935, cuando le contó que estaba preparando un folleto sobre las últimas publicaciones que había ido descubriendo en los últimos tiempos.

De 1968 conservamos una valoración más crítica de toda su obra (Galino Carrillo, 1968, pp. 1346-1372): "Frente al ideal krausista de cultura-dice allí-, que pretenden conseguir Francisco Giner de los Ríos y la Institución libre de enseñanza, Rufino Blanco desarrolla su programa docente en la escuela superior del magisterio sin claudicar de su fe católica". Por su parte Mélida-Nicolich lo sitúa dentro del regeneracionismo que se caracteriza "por su carácter católico y se oponen, por tanto, a la secularización de la vida pública y a la neutralidad religiosa en la escuela" (Mérida-Nicolich, 1994, pp. 621-624). García González (2008, pp. 1-4) lo sitúa en "la corriente «católica» (Manjón, Ruiz Amado, Rufino Blanco, Pedro Poveda...) de fundamentación neoescolástica que, a partir de segundo tercio del siglo XIX, surge como reacción a las ideas filosóficas de la época". Lo que nos queda sin embargo es el ejemplo del día a día. En el discurso de respuesta del académico de número Juan Zaragüeta y Bengoechea, discípulo predilecto del pedagogo, se preguntaba: “¿Qué es la vida de don Rufino Blanco sino un continuo rendimiento de pleitesía a tan altos objetivos, un ejemplar admirable de esfuerzo volitivo, una laboriosidad sin tregua?".

Víctor García Hoz resumía (1997, pp. 87-109): "las múltiples facetas de la vida de Rufino Blanco, honesta y pacientemente servidas, en su labor en el sistema escolar y sus aportaciones a la construcción y diseminación de los conocimientos y la ciencia pedagógica, permiten ver su figura como maestro de escuela; bibliógrafo; sistematizador; difusor de ideas y político; cristiano comprometido". Sobre este último aspecto, señala que "en todos los estudios sobre Rufino Blanco y las manifestaciones de quienes le conocieron y trataron, se menciona explícitamente su recio carácter cristiano". Reproduce así las citadas palabras de Menéndez y Pelayo que hacía suyas en 1907: "En reglas de lógica y en ley de hombre honrado y de creyente sincero tengo obligación de hacerlo". Su talante no será polémico ni combativo en el sentido negativo de la expresión. Sobre los trabajos de Giner de los Ríos y sus seguidores, a pesar de no poder compartir los presupuestos filosóficos, reconoce que "han realizado 
una meritoria labor de difusión del movimiento pedagógico internacional en España"12.

"Su aprecio por el valor pedagógico de las ideas cristianas -concluía García Hoz- se halla implícito en toda su obra, y no faltan manifestaciones explícitas", de las que cita algunas: "En síntesis, entiende que la ciencia y la crítica, en cuanto que servicios de la verdad y de la justicia, tienen sentido en que son «servicio de Dios», criterio último para juzgar el interés que puede tener cualquier obra pedagógica, ya sea un trabajo importante, ya sea un en apariencia insignificante folleto. La vida profesional y personal de Rufino Blanco y Sánchez fue ciertamente un continuado servicio a Dios. Su muerte en manos de la furia revolucionaria de la guerra civil española vino a dar un claro testimonio del carácter radicalmente cristiano de su vida y de sus ideas". Un hombre honrado y trabajador, buen cristiano, que entregó su vida a la enseñanza y la pedagogía, y que no dudó en hacer valer los valores católicos en la vida pública. En las circunstancias actuales, esta figura puede de servir de inspiración para entender la presencia de las ideas religiosas en la educación.

En resumen, podemos ver cómo la figura de Rufino Blanco y Sánchez no solo representa un testimonio histórico, sino que también su figura puede tener actualidad en el momento presente. En su labor educativa destacan las continuas iniciativas en la escuela pública, que le llevarán a experimentar con su propia actividad formativa. Fruto de todos estos trabajos en el aula, serán una larga serie de investigaciones donde combina la erudición con la experiencia. Toda esta labor le llevará a contactar con la ciencia elaborada en otros países, que intentará trasplantar a las realidades presentes en el nuestro. Imbuido por el espíritu regeneracionista, actuará convencido de que la educación es el mejor recurso con que cuenta la ciudadanía. Su espíritu inquieto e incansable le

\footnotetext{
${ }^{12}$ A su vez García Hoz establece tres facetas que le sitúan como cristiano comprometido: "su labor como publicista católico; su participación en instituciones y actividades católicas; sus relaciones con instituciones o personas dedicadas a la defensa de las ideas y la vida cristiana". Sobre la primera faceta como profesor e investigador lo considera en 1907 como "un servicio de Dios". Ahí están también El catecismo cíclico de la doctrina cristiana o las Joyas del catequista. Respecto a su colaboración con instituciones católicas, destaca la Institución del Divino maestro, "obra dedicada a la formación de maestros cristianos" creada por el obispo de Madrid, don Leopoldo Eijo y Garay. Recuerda además su puesto de presidente de los Cruzados de la enseñanza, fundada por el mismo prelado, así como su amistad con el padre Manjón, "con quien tuvo relaciones de verdadera amistad".
} 
llevó a internarse también en el mundo de la política-siempre vinculada a su condición de pedagogo- y del periodismo, donde destacó tanto como articulista como director y gestor de distintas publicaciones. Como persona destaca su ciencia y su afabilidad al mismo tiempo, propios de un maestro, tal como quiso verse siempre: como "un maestro de escuela". Como cristiano defendió la presencia de la religión en la escuela, hecho que posiblemente le comportó la muerte violenta al comienzo de la guerra civil española. En definitiva, una personalidad bastante completa que, si bien los conocimientos alcanzados mantienen cierta vigencia, también es cierto que deberían ser actualizados. En cualquier caso, un ejemplo y un modelo también para nuestros días.

\section{BiBLIOGRAFÍA:}

Arias Corrales, J., Aguirre Prado, L. y Pérez-Dario Zori, V. (1961). Un educador: D. Rufino Blanco y Sánchez. Madrid: Servicio.

BARRera, F. de P. (1916). Alcarreños ilustres: Rufino Blanco. Guadalajara: La Palanca (9/1/1916). Recuperado de: https://www.racmyp.es/docs/RufinoBlanco/AO6.pdf

Blanco, R. (1961). Rufino Blanco y Sánchez. En el centenario de su nacimiento.

Blanco Osborne, A. Reseña en Real Academia de la Historia. Recuperado de: http://dbe.rah.es/biografias/24421/rufino-blanco-y-sanchez.

Blanco Osborne, A. (2013). Rufino Blanco y Sánchez: una vida para pensar y educar. Algunos textos escogidos de Rufino Blanco. Isidora: revista de estudios galdosianos 21, pp. 133-140.

Blanco y SÁnchez, R. (1939). Fundamentos de educación moral y de educación cívica. Discurso leído por el Exmo. Sr. D. Rufino Blanco y Sánchez en el acto de recepción como académico de número el día 29 de marzo de 1939 y contestación el Ilmo. Sr. D. Juan Zaragüeta y Bengoechea, académico de número. Madrid: Luz y vida, 139 pp.

Boletín de Educación de Zaragoza (1937). 4 (1-2), p. 19.

Boletín provincial de Educación de Pontevedra (1937). (1), pp. 15 y 16.

Corts Giner, M. I. (1986). Rufino Blanco y Sánchez, pionero de los estudios bibliográficos-pedadógicos en España. Cuestiones pedagógicas: Revista de 
ciencias de la educación, 53-58. Recuperado de: https://www.racmyp.es/ docs/RufinoBlanco/ARB7.pdf.

Boletín de la Institución Teresiana (1935). D. Rufino Blanco y Sánchez (241), p. 115.

Galino Carrillo, M. A. (1968). Rufino Blanco y Sánchez. Textos pedagógicos hispanoamericanos (pp. 1346-1372). Madrid: Narcea.

García GonzÁlez, F. (2008). Rufino Blanco y Sánchez (1861-1936). El Catón. Revista de Investigación y Difusión Cultural del Museo del Niño. Sección Pedagogos de Castilla-La Mancha 14, 1-4. Recuperado de: https://www. racmyp.es/docs/RufinoBlanco/ARB6.pdf.

García Hoz, V. (1997). Biografía de D. Rufino Blanco y Sánchez. Académicos vistos por académicos: juristas y filósofos (pp. 87-109). Recuperado de: https://www.racmyp.es/docs/varios/V1-5.pdf.

Garrido Palacios, M. (1979). Historia de la Educación en España (1857-1975). Una visión hacia lo local, en Reloj de arena. Historia de la Educación en España (1857-1975) (pp. 89-95). Cabra del Santo Cristo: Contraluz.

Getino, L. (1917). Caracteres y siluetas: D. Rufino Blanco. Madrid: Rosas y Espinas.

Gómez Blanco, F. Testimonio al autor (8/4/2020).

González Blanco, M. Testimonio al autor (8/4/2020).

Homenaje a D. Rufino Blanco (1932). La Palanca (1109:6.1.1932), p. 1.

Martorell Linares, M. (2002). De ciencias sociales y ángeles custodios: la Real Academia de Ciencias Morales y Políticas bajo la guerra y la autarquía. Historia y Política 8, 229-252.

MéridA-Nicolich, E. (1994). Rufino Blanco y Sánchez. Historia de la educación en España y América. En Delgado Criado, B. (ed.). La educación en la España contemporánea (1789-1975), III, Madrid: Fundación de Santa María, pp. 621-624.

Navarro, M. (1961). La vida ejemplar de D. Rufino Blanco. Recuperado de: https:/www.racmyp.es/contenido/archivo-rufino-blanco/46/

Torrebadella-Flix, X. (2014). Rufino Blanco Sánchez y las fuentes bibliográficas de la educación física y el deporte en España. International Journal of Sport Science 37, 281. Recuperado de: https://cutt.ly/bnQjK0v 
Rogerio SÁnchez, J. et al. (1933). Homenaje a D. Rufino Blanco y Sánchez con motivo de su jubilación. Madrid. Recuperado de: https://www.racmyp. es/docs/RufinoBlanco/AO21.pdf

Vida Económica (1935). Don Rufino Blanco (908), p. 125. Recuperado de: https://www.racmyp.es/docs/RufinoBlanco/AO23.pdf

Zahonero, J. (1916). Rufino Blanco y su nueva obra. La Independencia (16/03/1916). Recuperado de: https://www.racmyp.es/docs/RufinoBlanco/ AO23.pdf

Zaragüeta y Bengoechea, J. (1961) Necrología de Rufino Blanco en su primer centenario. Anales de la Real Academia de Ciencias Morales y Politicas, 243-252. Recuperado de: https://www.racmyp.es/docs/RufinoBlanco/ ARB4.pdf 